This copy is for your personal, non-commercial use only.

If you wish to distribute this article to others, you can order high-quality copies for your colleagues, clients, or customers by clicking here.

Permission to republish or repurpose articles or portions of articles can be obtained by following the guidelines here.

The following resources related to this article are available online at www.sciencemag.org (this information is current as of April 16, 2013 ):

Updated information and services, including high-resolution figures, can be found in the online version of this article at:

http://www.sciencemag.org/content/337/6094/554.full.html

Supporting Online Material can be found at:

http://www.sciencemag.org/content/suppl/2012/07/05/science.1222779.DC1.html

A list of selected additional articles on the Science Web sites related to this article can be found at:

http://www.sciencemag.org/content/337/6094/554.full.html\#related

This article cites 29 articles, 6 of which can be accessed free:

http://www.sciencemag.org/content/337/6094/554.full.html\#ref-list-1

This article has been cited by 4 articles hosted by HighWire Press; see:

http://www.sciencemag.org/content/337/6094/554.full.html\#related-urls

This article appears in the following subject collections:

Astronomy

http://www.sciencemag.org/cgi/collection/astronomy 


\section{Radio Detections During Two State Transitions of the Intermediate-Mass Black Hole HLX-1}

Natalie Webb, ${ }^{1,2 *}$ David Cseh, ${ }^{3}$ Emil Lenc, ${ }^{4,5}$ Olivier Godet, ${ }^{1,2}$ Didier Barret, $^{1,2}$ Stephane Corbel, $^{3}$ Sean Farrell, ${ }^{5,6}$ Robert Fender, ${ }^{7}$ Neil Gehrels, ${ }^{8}$ Ian Heywood ${ }^{9}$

Relativistic jets are streams of plasma moving at appreciable fractions of the speed of light. They have been observed from stellar-mass black holes ( 3 to 20 solar masses, $M_{\odot}$ ) as well as supermassive black holes $\left(\sim 10^{6}\right.$ to $\left.10^{9} \mathrm{M}_{\odot}\right)$ found in the centers of most galaxies. Jets should also be produced by intermediate-mass black holes $\left(\sim 10^{2}\right.$ to $\left.10^{5} M_{\odot}\right)$, although evidence for this third class of black hole has, until recently, been weak. We report the detection of transient radio emission at the location of the intermediate-mass black hole candidate ESO 243-49 HLX-1, which is consistent with a discrete jet ejection event. These observations also allow us to refine the mass estimate of the black hole to be between $\sim 9 \times 10^{3} \mathrm{M}_{\odot}$ and $\sim 9 \times 10^{4} \mathrm{M}_{\odot}$.

$\mathrm{I}$ thas been proposed that the kinetic power output of any black hole is only related to the mass accreted [in Eddington units (1)] onto it and does not depend on the mass of the black hole itself (2). If this is true, jet emission, which is most frequently detected through radio emission, is to be expected not only from stellarmass black holes and supermassive black holes, but from black holes of all masses. This includes ultraluminous x-ray sources (ULXs), which are non-nuclear extragalactic $\mathrm{x}$-ray point sources that exceed the Eddington luminosity (where the radiation force is balanced by the gravitational force) for a stellar-mass black hole. These could be stellar-mass black holes undergoing hyperaccretion $(3,4)$ and/or beaming $(5,6)$. Alternatively, they could contain black holes of a slightly higher mass, between 30 and 90 solar masses $\left(M_{\odot}\right)(7)$, or could be the missing class of intermediate-mass black holes (8).

To date, no variable radio emission associated with jets has been detected from ultraluminous $\mathrm{x}$-ray sources, despite numerous observing campaigns $(9,10)$. By contrast, nonvarying nebulalike extended radio emission, which is likely to be powered by the central black hole, has been detected around some ULXs (11-13). ESO 24349 HLX-1 (hereafter referred to as HLX-1) not only is a ULX but is currently the best intermediatemass black hole candidate. If HLX-1 harbors an intermediate-mass black hole, it accretes at comparable fractions of the Eddington luminosity, as stellar-mass black holes in binaries (14). Hence, HLX-1 is expected to display similarities to the latter class of objects. In that respect, spectral state transitions reminiscent of black hole binaries have already been reported $(15,16)$. It is therefore the ideal object in which to search for jet emission, so as to verify the scale invariance of jets from black holes.

HLX-1 was detected serendipitously by XMMNewton on 23 November 2004 in the outskirts of the edge-on spiral galaxy ESO 243-49, 8 arc sec from the nucleus (17). The distance to HLX-1 measured from its $\mathrm{H}_{\alpha}$ emission line confirms that ESO 243-49 is the host galaxy (18). HLX-1 therefore has a maximum unabsorbed $\mathrm{x}$-ray luminosity, assuming isotropic emission, of $1.1 \times 10^{42}$

${ }^{1}$ Université de Toulouse; UPS-OMP; IRAP, F-31028 Toulouse, France. ${ }^{2}$ CNRS; IRAP; 9 avenue du Colonel Roche, BP 44346, F-31028 Toulouse Cedex 4, France. ${ }^{3}$ Laboratoire Astrophysique des Interactions Multi-echelles (UMR 7158), CEAIDSM-CNRSUniversité Paris Diderot, CEA Saclay, F-91191 Gif-sur-Yvette, France. ${ }^{4}$ Australia Telescope National Facility, CSIRO Astronomy and Space Science, PO Box 76, Epping, NSW 1710, Australia. ${ }^{5}$ Sydney Institute for Astronomy, School of Physics, University of Sydney, Sydney, NSW 2006, Australia. 'Department of Physics and Astronomy, University of Leicester, Leicester LE1 7RH, UK. ${ }^{7}$ School of Physics and Astronomy, University of Southampton Highfield, Southampton S017 1B], UK. ${ }^{8}$ Astroparticle Physics Laboratory, NASA/Goddard Space Flight Center, Greenbelt, MD 20771, USA. 'Department of Physics, University of Oxford, Oxford OX1 3RH, UK.

*To whom correspondence should be addressed. E-mail: natalie.webb@irap.omp.eu

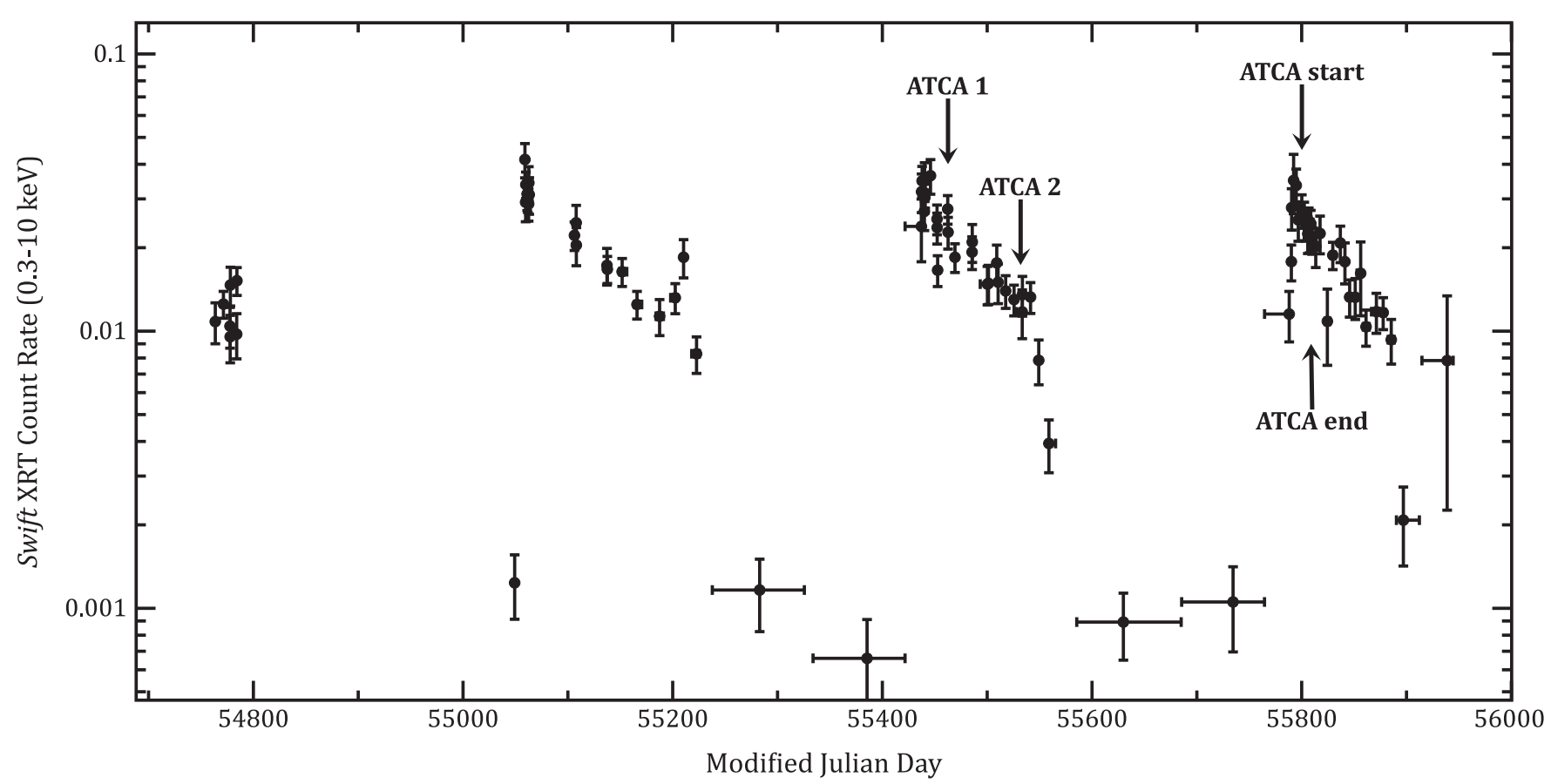

Fig. 1. Long-term Swift x-ray telescope light curve showing the dates of the first two ATCA observations and the period during which the subsequent five ATCA observations were taken. Three $x$-ray state transitions from the low/hard state (count rate less than $\sim 0.002,0.3$ to $10.0 \mathrm{keV}$ ) to the high/soft state (count rate between $\sim 0.01$ and $\sim 0.05,0.3$ to $10.0 \mathrm{keV}$ ) can be seen. 


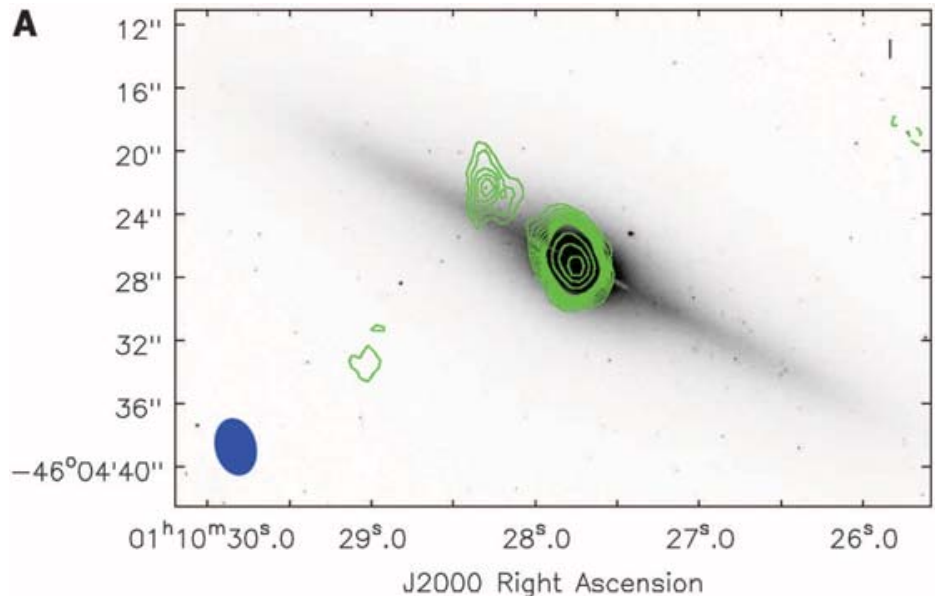

Fig. 2. (A) Five- and 9-GHz combined radio observations [contours: $-3,3$, $4,5,6,7,8,9,10,15,20$, and 25 times the $1 \sigma$ root mean square (RMS) noise level $(5.6 \mu \mathrm{Jy}$ per beam)] using the radio data taken on 13 September 2010, 31 August 2011, and 3 and 4 September 2011 with ATCA and superimposed on an I-band Hubble Space Telescope image of ESO 243-49 (inverted color map). The beam size is shown at the lower left. The galaxy, ESO $243-49$, is clearly detected in radio. An $8 \sigma$ point source falls at RA = $01^{\mathrm{h}} 10^{\mathrm{m}} 28.28^{\mathrm{s}}$ and $\mathrm{dec}=-46^{\circ} 04^{\prime} 22.3^{\prime \prime}$ ( $1 \sigma$ error on the position of RA $=$

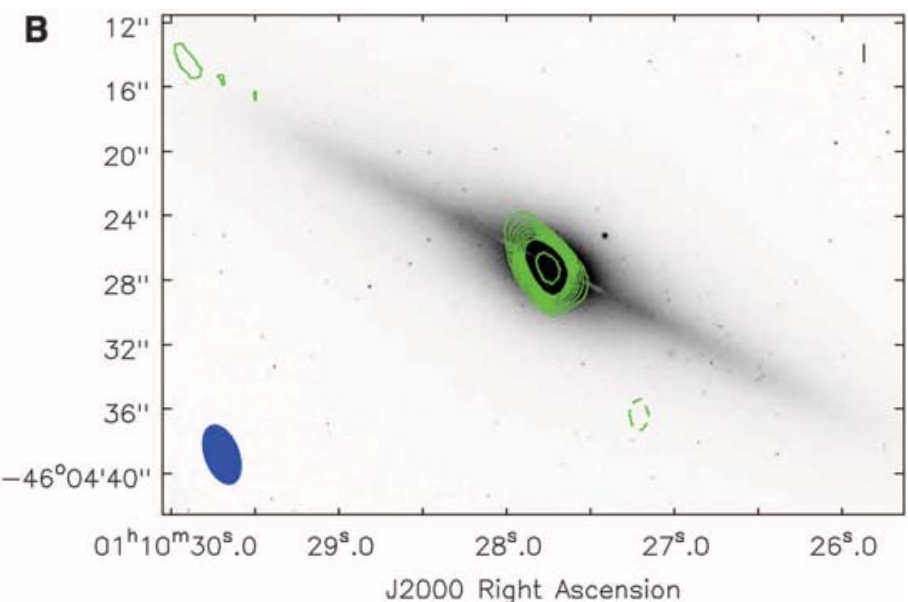

$0.43^{\prime \prime}$ and dec $=0.67^{\prime \prime}$ ), well within the 0.3" Chandra error circle of HLX-1. (B) Five- and 9-GHz combined radio observations [contours: $-3,3,4,5,6,7$, $8,9,10,15,20$, and 25 times the $1 \sigma$ RMS noise level $(7.0 \mu$ ]y per beam)] made from the 3 December 2010, 25 August 2011, and 1 September 2011 ATCA observations and superimposed on the same I-band Hubble Space Telescope image of ESO 243-49. The galaxy ESO 243-49 is again clearly detected, but no source is found within the Chandra error circle. The beam size is shown at the lower left. ergs $\mathrm{s}^{-1}$ (17). HLX-1 qualifies as a ULX because of the non-nuclear situation of this point source and because it exceeds the Eddington luminosity for a stellar-mass black hole by three orders of magnitude. From the x-ray luminosity and the conservative assumption that this value exceeds the Eddington limit by at most a factor of 10 (3), a lower limit of $500 M_{\odot}$ was derived for the mass of the black hole (17). The maximum mass, however, is not constrained. The x-ray to optical flux ratio $(17,19)$ is far greater than expected from an active galactic nucleus, but without an estimate of this maximum mass, it could be argued that HLX-1 is a non-nuclear supermassive black hole [e.g., (20)].

We observed HLX-1 with the Australia Telescope Compact Array (ATCA) in the 750-m configuration on 13 September 2010 (21), when regular X-ray monitoring of HLX-1 with the Swift satellite (22) showed that HLX-1 had just undergone a transition from the low/hard $\mathrm{x}$-ray state to the high/soft x-ray state. This transition occurs for HLX-1 when the count rate increases by more than a factor of 10 in just a few days (Fig. 1) $(16,23)$. Galactic black hole binaries are known to regularly emit radio flares around the transition from the low/hard to the high/soft state [e.g., $(24,25)]$. Such flares are associated with ejection events; for example, an expelled jet can lead to radio flaring when the highervelocity ejecta collides with the lower-velocity material produced by the steady jet. As well as detecting radio emission from the nucleus of the galaxy, we detected a radio point source at right ascension $(\mathrm{RA})=01^{\mathrm{h}} 10^{\mathrm{m}} 28.28^{\mathrm{s}}$ and declination $(\mathrm{dec})=-46^{\circ} 04^{\prime} 22.3^{\prime \prime}$, coincident with the Chandra x-ray position of HLX-1 (26). Combining the 5-GHz and 9-GHz data gives a detection of $50 \mu \mathrm{Jy}$ per beam and a $1 \sigma$ noise level of $11 \mu \mathrm{Jy}$, and thus

Table 1. The seven radio observations organized by date. The second column shows the Swift x-ray unabsorbed flux ( 0.5 to $10.0 \mathrm{keV}$ ), with $90 \%$ confidence intervals in parentheses. The third column shows the combined $5-$ and $9-\mathrm{GHz}$ peak brightness radio flux (with the associated $1 \sigma$ noise level) for the detections, or the $3 \sigma$ upper limit for the nondetections. The final column gives the radio flux from fitting a point source (with the associated $1 \sigma$ noise level) using the point spread function.

\begin{tabular}{|c|c|c|c|}
\hline Observation date & $\begin{array}{c}\text { X-ray flux } \\
\text { (ergs } \mathrm{cm}^{-2} \mathrm{~s}^{-1} \text { ) }\end{array}$ & $\begin{array}{c}5+9 \mathrm{GHz} \text { peak flux } \\
(\mu \mathrm{Jy} / \text { beam })\end{array}$ & $5+9 \mathrm{GHz}$ flux density $(\mu \mathrm{Jy})$ \\
\hline 13 Sep 2010 & $4.57\left({ }_{-0.50}^{+0.68}\right) \times 10^{-13}$ & $50(11)$ & $42(10)$ \\
\hline 3 Dec 2010 & $2.40\left(\begin{array}{l}+0.60 \\
-0.50\end{array}\right) \times 10^{-13}$ & $<36$ & $11(20)$ \\
\hline 25 Aug 2011 & $4.57( \pm 0.30) \times 10^{-13}$ & $<30$ & $14.5(7)$ \\
\hline 31 Aug 2011 & $4.57( \pm 0.30) \times 10^{-13}$ & $51(10)$ & $63(18)$ \\
\hline 1 Sep 2011 & $4.57( \pm 0.30) \times 10^{-13}$ & $<31$ & $25(10.5)$ \\
\hline 3 Sep 2011 & $4.57( \pm 0.30) \times 10^{-13}$ & $45(10.5)$ & $43(10)$ \\
\hline 4 Sep 2011 & $4.57( \pm 0.30) \times 10^{-13}$ & $30(7.5)$ & $27(7.5)$ \\
\hline
\end{tabular}

a $4.5 \sigma$ detection at the position of HLX-1, at a time when such emission can be expected (Fig. 2A and Table 1).

The radio flares in galactic black hole binaries are typically brighter than the nonflaring radio emission by a factor of 10 to $>100(9)$ and generally last one to several days [e.g., XTE $\mathrm{J} 1859+226$ (27)]. Once the high/soft state has been achieved, the core jet is suppressed [e.g., (24)]. To determine whether the radio emission that we detected was transient and thus associated with a radio flare, we made another observation with ATCA in the 6-km configuration on 3 December 2010, when HLX-1 was declining from the high/ soft state and when no flaring was expected. This observation again showed emission from the nucleus of the galaxy, consistent with that of the previous radio observation, but revealed no source at the position of HLX-1. The $3 \sigma$ nondetection for the combined 5- and 9-GHz data is $36 \mu \mathrm{Jy}$ per beam (Fig. 2B and Table 1). These observations suggest that the source is variable.
To confirm the variability, we reobserved HLX-1 when it had just undergone another transition from the low/hard x-ray state to the high/ soft x-ray state in August 2011 (Fig. 1). All five of the 2011 observations (Table 1) were made in a similar configuration to the December 2010 observation. We observed three noncontiguous detections $(\geq 4 \sigma)$ and two noncontiguous nondetections of the source (Table 1). This indicates that two flares were detected during this period.

To determine whether the source was indeed variable, we fitted each observation using a point source, with the point spread function. We used the position of HLX-1 when the source was not detected. This allowed us to estimate the flux and the associated errors (Table 1) even for a nondetection. We tested whether the data could be fitted with a constant - namely, the mean of the data. We used a chi-square test to compare these data to the mean flux value and found a reduced chi-square $\left(\chi_{v}^{2}\right)$ value of 2.5 (5 degrees of freedom); because this is much greater than unity, 
a constant is a poor fit to the data and hence the variable nature of the phenomenon is supported. Further, combining all of the detections ( 5 and $9 \mathrm{GHz}$ ), the source was observed at $45 \mu \mathrm{Jy}$ per beam, with a $1 \sigma$ noise level of $5.5 \mu \mathrm{Jy}$; this represents a confident detection at the $8 \sigma$ level. Combining in a similar fashion the data in which no radio emission was detected, we obtained a $3 \sigma$ upper limit in the combined 5- and 9-GHz data of $21 \mu \mathrm{Jy}$ per beam (Fig. 2). The variability rules out emission from a nebula. Hence, the observed variable radio emission is again consistent with a transient jet ejection event.

Observations of supermassive black holes and stellar-mass black holes have supported the scale invariance of jets $(28,29)$ through comparing x-ray and radio measurements - tracers of mass accretion rate and kinetic output, respectively - with the associated black hole masses to form a "fundamental plane of black hole activity" (28). Under the hypothesis that HLX-1 is indeed an intermediatemass black hole, we can test the proposed relation. We take what is generally considered to be the maximum mass of intermediate-mass black holes, $\sim 1 \times 10^{5} M_{\odot}(8)$, and the x-ray luminosity, $5.43 \times 10^{41} \mathrm{ergs} \mathrm{s}^{-1}(0.5$ to $10.0 \mathrm{keV})$, determined from Swift x-ray telescope (30) observations made at the same time as our radio detection. Continuum (nonflaring) radio emission can then be estimated with the aforementioned relationship (29), which is based on a sample that includes black holes in all different $\mathrm{x}$-ray states. This relation implies a continuum radio emission at the $\sim 20 \mu \mathrm{Jy}$ level. This is slightly lower than the $3 \sigma$ nonflaring upper limit, which suggests that the mass of the black hole is likely to be less than $\sim 1 \times 10^{5} M_{\odot}$.

Radio flares are seen to occur in galactic black hole binaries when the x-ray luminosity is 10 to $100 \%$ of the Eddington luminosity (14). HLX-1 has already shown behavior similar to that of galactic black hole binaries. Therefore, if we assume that the radio flares that we observed also occur when the x-ray luminosity is 10 to $100 \%$ of the Eddington luminosity, then the black hole mass would be between $\sim 9.2 \times 10^{3} M_{\odot}$ and $\sim 9.2 \times$ $10^{4} M_{\odot}$, commensurate with the mass estimate above and those of $(16,23,31)$ and confirming the intermediate-mass black hole status.

\section{References and Notes}

1. The mass accreted $(\dot{m})$ in Eddington units is represented by a dimensionless ratio, $\dot{m}=\dot{M} / \dot{M}_{\mathrm{EDD}}$, where $M$ is the accretion rate and $\dot{M}_{\text {EDD }}$ is the maximum rate of accretion due to the Eddington luminosity.

2. S. Heinz, R. A. Sunyaev, Mon. Not. R. Astron. Soc. 343, 159 (2003).

3. M. C. Begelman, Astrophys. J. 568, L97 (2002).

4. J. C. Gladstone, T. P. Roberts, C. Done, Mon. Not. R. Astron. Soc. 397, 1836 (2009).

5. A. R. King, M. B. Davies, M. J. Ward, G. Fabbiano, M. Elvis, Astrophys. J. 552, L109 (2001).

6. E. Körding, H. Falcke, S. Markoff, Astron. Astrophys. 382 L13 (2002).

7. L. Zampieri, T. P. Roberts, Mon. Not. R. Astron. Soc. 400 677 (2009).

8. M. C. Miller, E. J. M. Colbert, Int. J. Mod. Phys. D 13, 1 (2004).

9. E. Körding, E. Colbert, H. Falcke, Astron. Astrophys. 436, 427 (2005).
10. M. Freeland, Z. Kuncic, R. Soria, G. V. Bicknell, Mon. Not. R. Astron. Soc. 372, 630 (2006)

11. P. Kaaret, S. Corbel, A. H. Prestwich, A. Zezas, Science 299, 365 (2003).

12. N. A. Miller, R. F. Mushotzky, S. G. Neff, Astrophys. J. 623, L109 (2005)

13. D. Cseh et al., Astrophys. J. 749, 17 (2012).

14. R. P. Fender, T. M. Belloni, E. Gallo, Mon. Not. R. Astron. Soc. 355, 1105 (2004).

15. O. Godet, D. Barret, N. A. Webb, S. A. Farrell, N. Gehrels, Astrophys. J. 705, L109 (2009).

16. M. Servillat et al., Astrophys. J. 743, 6 (2011).

17. S. A. Farrell, N. A. Webb, D. Barret, O. Godet, ]. M. Rodrigues, Nature 460, 73 (2009).

18. K. Wiersema et al., Astrophys. J. 721, L102 (2010).

19. R. Soria et al., Mon. Not. R. Astron. Soc. 405, 870 (2010).

20. J. Guedes, P. Madau, M. Kuhlen, J. Diemand, M. Zemp, Astrophys. J. 702, 890 (2009).

21. See supplementary materials on Science Online.

22. N. Gehrels et al., Astrophys. J. 611, 1005 (2004).

23. O. Godet et al., http://arxiv.org/abs/1204.3461 (2012).

24. R. P. Fender, J. Homan, T. M. Belloni, Mon. Not. R. Astron. Soc. 396, 1370 (2009)

25. S. Corbel, R. P. Fender, J. A. Tomsick, A. K. Tzioumis, S. Tingay, Astrophys. J. 617, 1272 (2004).

26. N. A. Webb et al., Astrophys. J. 712, L107 (2010).

27. C. Brocksopp et al., Mon. Not. R. Astron. Soc. 331, 765 (2002).
28. A. Merloni, S. Heinz, T. di Matteo, Mon. Not. R. Astron. Soc. 345, 1057 (2003).

29. E. Körding, H. Falcke, S. Corbel, Astron. Astrophys. 456, 439 (2006)

30. D. N. Burrows et al., Space Sci. Rev. 120, 165 (2005).

31. S. W. Davis et al., Astrophys. J. 734, 111 (2011).

Acknowledgments: Supported by the European Community's Seventh Framework Programme (FP7/2007-2013) under grant agreement number ITN 215212 "Black Hole Universe" (D.C. and S.C.), the UK Science and Technology Facilities Council (S.F.), and an Australian Research Council postdoctoral fellowship funded by grant DP110102889 (S.F.). The Australia Telescope is funded by the Commonwealth of Australia for operation as a national facility managed by CSIRO; the data can be accessed at http://atoa.atnf.csiro.au/query.jsp. This work made use of data supplied by the UK Swift Science Data Centre at the University of Leicester (www.swift.ac.uk/swift_portal).

\section{Supplementary Materials}

www.sciencemag.org/cgi/content/full/science.1222779/DC1

Materials and Methods

Table S1

References $(32,33)$

April 2012; accepted 11 June 2012

Published online 5 July 2012

10.1126/science.1222779

\section{Kepler-36: A Pair of Planets with Neighboring Orbits and Dissimilar Densities}

Joshua A. Carter, ${ }^{1}{ } \dagger$ Eric Agol, ${ }^{2}{ }{ } \dagger$ William J. Chaplin, ${ }^{3}$ Sarbani Basu, ${ }^{4}$ Timothy R. Bedding, ${ }^{5}$ Lars A. Buchhave, ${ }^{6}$ Jørgen Christensen-Dalsgaard, ${ }^{7}$ Katherine M. Deck, ${ }^{8}$ Yvonne Elsworth, ${ }^{3}$ Daniel C. Fabrycky, ${ }^{9}$ Eric B. Ford, ${ }^{10}$ jonathan J. Fortney, ${ }^{11}$ Steven J. Hale, ${ }^{3}$ Rasmus Handberg, ${ }^{7}$ Saskia Hekker, ${ }^{12}$ Matthew J. Holman ${ }_{1}^{13}$ Daniel Huber, ${ }^{14}$ Christopher Karoff, ${ }^{7}$ Steven D. Kawaler, ${ }^{15}$ Hans Kjeldsen, ${ }^{7}$ Jack J. Lissauer, ${ }^{14}$ Eric D. Lopez, ${ }_{1}^{11}$ Mikkel N. Lund, ${ }^{7}$ Mia Lundkvist, ${ }^{7}$

Travis S. Metcalfe, ${ }^{16}$ Andrea Miglio, ${ }^{3}$ Leslie A. Rogers, ${ }^{8}$ Dennis Stello, ${ }^{5}$ William J. Borucki, ${ }^{14}$ Steve Bryson, ${ }_{14}$ Jessie L. Christiansen, ${ }_{17}$ William D. Cochran, ${ }_{1}^{18}$ John C. Geary, ${ }_{13}^{13}$ Ronald L. Gilliland, ${ }^{19}$ Michael R. Haas, ${ }^{14}$ Jennifer Hall, ${ }^{20}$ Andrew W. Howard, ${ }^{21}$ Jon M. Jenkins, ${ }^{17}$ Todd Klaus, $^{20}$ David G. Koch, ${ }^{14}$ David W. Latham, ${ }^{13}$ Phillip J. MacQueen, ${ }^{18}$ Dimitar Sasselov, ${ }^{13}$ Jason H. Steffen, ${ }^{22}$ Joseph D. Twicken, ${ }^{17}$ Joshua N. Winn ${ }^{8}$

In the solar system, the planets' compositions vary with orbital distance, with rocky planets in close orbits and lower-density gas giants in wider orbits. The detection of close-in giant planets around other stars was the first clue that this pattern is not universal and that planets' orbits can change substantially after their formation. Here, we report another violation of the orbitcomposition pattern: two planets orbiting the same star with orbital distances differing by only $10 \%$ and densities differing by a factor of 8 . One planet is likely a rocky "super-Earth," whereas the other is more akin to Neptune. These planets are 20 times more closely spaced and have a larger density contrast than any adjacent pair of planets in the solar system.

$\mathrm{T}$ he detection of the first "hot Jupiter" around a Sun-like star (I) was surprising to researchers who based their expectations on the properties of the solar system. Theorists soon developed models of planetary "migration" to explain how the planets' orbits can shrink (2). This work led to a broader recognition that the architecture of planetary systems can change substantially after their formation. The planetary system reported in this paper is another example of an "extreme" planetary system that will serve as a stimulus to theories of planet migration and orbital rearrangement. The system features two planets in neighboring orbits with substantially different compositions.

This system was detected from the miniature eclipses or transits that cause the host star to appear fainter when the planets pass in front of the star. The target star (Kepler Object of Interest 277; hereafter, Kepler-36; also KIC 11401755, 2MASS $19250004+4913545$ ) is one of about 150,000 stars that is subject to nearly continuous photometric surveillance by the Kepler spacecraft (3-5). The Kepler data revealed the transits of two planets. The loss of light during transits by planet $b$ is only $17 \%$ as large as that from planet c 